\title{
THE OSTEOCUTANEOUS FREE FIBULAR GRAFT
}

\author{
D. H. HARRISON
}

\author{
From Mount Vernon Hospital, Northwood
}

\begin{abstract}
Free osteocutaneous fibular grafts, revascularised by microvascular anastomoses, have been used for one-stage reconstruction of extensive bone and skin loss in the lower leg in seven patients. The addition of an integral skin flap to a vascularised fibular graft makes reconstruction of bone defects with significant skin loss possible, and the technique for designing and raising such a flap is presented.

The advantages of this transfer over other microvascular osteocutaneous flaps are the available length of straight cortical bone, the large thin skin flap, the good diameter of the vascular pedicle and the fact that dissection is carried out under a tourniquet.
\end{abstract}

With the advent of vascularised bone grafting the success rate of replacing extensive areas of bone loss in long bones has been considerably increased. Conventional non-vascularised bone grafts undergo a sequence of necrosis and regeneration often called creeping substitution, but with microvascular techniques an intact circulation through the graft can be maintained, thus avoiding both the period of substitution and the bone atrophy and resorption which is otherwise inevitable. Revascularisation of the bone enhances the healing at each end of the graft and enables it to withstand possible infection. Moreover, should a fracture of the graft occur in the postoperative period, it will unite like any other long bone fracture (Haw, O’Brien and Kurata 1978).

In 1975, Taylor, Miller and Ham described two cases in which vascularised free fibular grafts had been used to treat severe fractures of the tibia and fibula, but their technique for harvesting the fibula was very timeconsuming. Gilbert (1979) and Tamai et al. (1980) refined the method of removing the fibula; reduction of the time for the operation to less than an hour made the technique much more applicable.

In severe injuries of the lower limb, however, when there is extensive bone damage or loss, the overlying skin is often similarly damaged. Standard free fibular grafts have no overlying skin flap and can be used only when the skin overlying the bone defect is intact, or after the earlier provision of a skin flap graft. A number of osteocutaneous flaps have been developed, such as the groin flap, the dorsalis pedis flap and the radial arm flap, but these can replace only short bony defects and osteocutaneous defects of $10 \mathrm{~cm}$ or more in length provide distinct problems.

D. H. Harrison. FRCS. Consultant Plastic Surgeon

The Plastic Surgery Centre. Mount Vernon Hospital, Northwood, Middlesex HA6 2RN. England

1 1986 British Editorial Society of Bone and Joint Surgery $0301620 \times 865157 \$ 2.00$
In 1978 Taylor and Watson described an osteocutaneous flap taken from the ilium and based on the deep circumflex iliac artery. This provides both cancellous and cortical bone and a fairly reliable skin flap, but the natural curvature of the ilium means that subperiosteal fracture of the bone graft is required in order to straighten it and even then it is unsuitable for the replacement of defects longer than $10 \mathrm{~cm}$. In addition, the overlying skin flap may be unreliable (particularly in fat patients), the osteosynthesis is not easy and, since muscle cutting is inevitable. the flap is a bloody one and may leave a hernia through the bone defect. One alternative is a vascularised free rib graft as first described by Buncke et al. in 1977, but they found that one rib was inadequate and fractured easily. A. R. Godfrey and B. Bailey (personal communication 1985) have recently described the use of two ribs with an overlying latissimus dorsi muscle flap, but again the curvature of the rib graft requires correction by subperiosteal fractures.

In 1983 Chen and Yan used a technique by which an overlying skin flap could be harvested with a free fibular graft and described two cases. We have used this technique to reconstruct seven extensive defects, all with tibial bone loss in excess of $10 \mathrm{~cm}$. The advantages include the length, shape and composition of the fibula from which up to $20 \mathrm{~cm}$ of straight cortical bone can be obtained. Osteosynthesis is easy since the fibular graft can be placed within the reamed medullary cavity of the tibia, rarely needing screw fixation, and the osteocutaneous graft can be dissected out under tourniquet control with virtually no blood loss.

\section{TECHNIQUE OF OPERATION}

Anatomy. The posterior tibial artery bifurcates deep to the soleus muscle giving off the peroneal artery which runs obliquely down towards the fibula and then along its medial crest beneath the flexor hallucis longus. As it passes close to the fibula it gives off nutrient vessels and musculocutaneous branches, also called circular arteries 


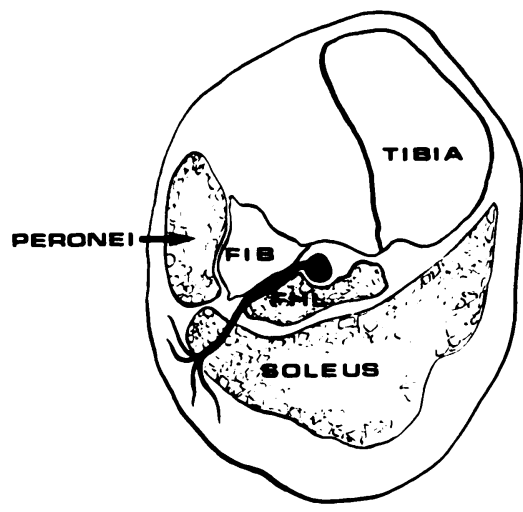

Fig. 1

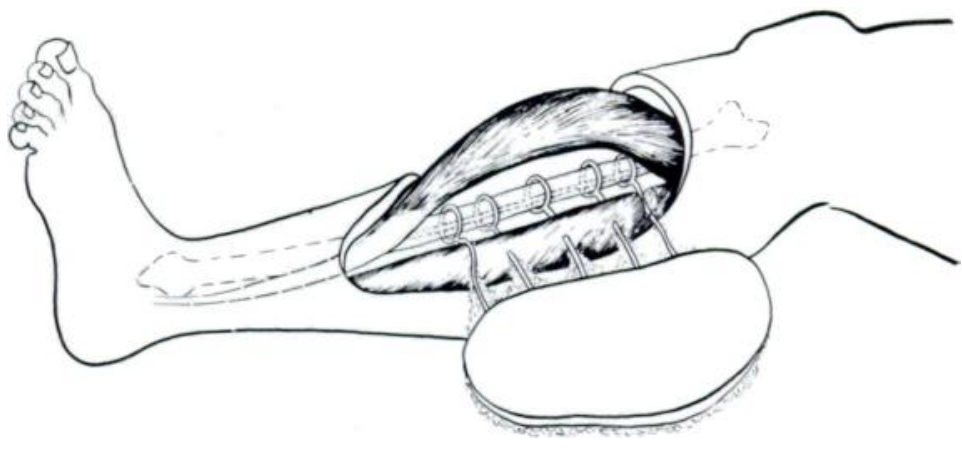

Fig. 2

Figure I Cross-section to show the relationships of the peroneal artery in mid-calf, with one of the cutaneous branches passing through the anterior edge of soleus to reach the deep fascia. Figure 2 Diagram to show the cutaneous branches of the peroneal artery penetrating the soleus and entering the skin flap.

(Fig. 1). Cutaneous branches, usually three in number, penetrate the soleus muscle near its origin from the fibula and run in its lateral edge. The upper and lower branches bifurcate to give five vessels which penetrate the deep fascia to enter the subcutaneous tissue and form a fascial plexus which supplies the overlying skin (Fig. 2). The area of the skin supplied by this network is approximately $10 \mathrm{~cm}$ in width based on the longitudinal line of the fibula.

The cutaneous vessels do not pass through the lateral intermuscular septum between the soleus and the peroneus longus, and it is therefore safe to use this plane to gain access to the vessels and to the anterior aspect of the fibula.

Dissection. A skin flap of the size required to resurface the defect is marked over the line of the fibula, with proximal and distal longitudinal extensions of the incision to allow deep access. The lateral popliteal nerve is located at the neck of the fibula and preserved. The dissection of the flap is deepened through the deep fascia, which is elevated with it. At the posterior edge of the flap the lateral belly of the gastrocnemius muscle is defined and in front of it the soleus is seen. Considerable care must be taken to find and preserve the perforating cutaneous vessels at the anterior edge of soleus. The muscle is split longitudinally $1 \mathrm{~cm}$ behind its anterior border in order to retain the five cutaneous branches with the flap.

The incision at the anterior edge of the flap is then deepened, again through the deep fascia, avoiding the musculocutaneous nerve inferiorly. The flap can then be elevated from the peroneus brevis and longus but again great care is needed to avoid cutting the cutaneous branches at the posterior aspect of the peroneus longus (Fig. 3).

The intermuscular septum between the peroneus longus and soleus is then exposed, leaving the cutaneous vessels clearly visible as they supply the flap through the previously freed anterior edge of soleus. The incision is deepened through the intermuscular septum and the peroneus longus is elevated from the anterior aspect of the fibula leaving the periosteum intact, preferably with a thin covering layer of muscle.

At this stage the fibula is divided proximally and distally allowing sufficient length to fill the bone defect with 1 to $2 \mathrm{~cm}$ extra at each end for insertion into the medullary cavity of the tibia. At the lower end the peroneal artery is seen emerging from the lower border of flexor hallucis longus and is divided. The lower $5 \mathrm{~cm}$ of the fibula is left intact to maintain the mortise of the ankle joint.

The fibula can then be lifted partially out of the wound and rotated to provide access to the interosseous membrane, which is divided from distal to proximal, allowing the osteocutaneous graft to be lifted further. Some fibres of flexor hallucis and the tibialis posterior are divided to free the bone. and at the proximal end the peroneal vascular pedicle comes into view. At this stage the tourniquet is released and it is possible to confirm that both the bony and the cutaneous elements of the flap are viable. The surgeon confirms that the vascular pedicle at the recipient site has been prepared and the flap vessels are then divided.

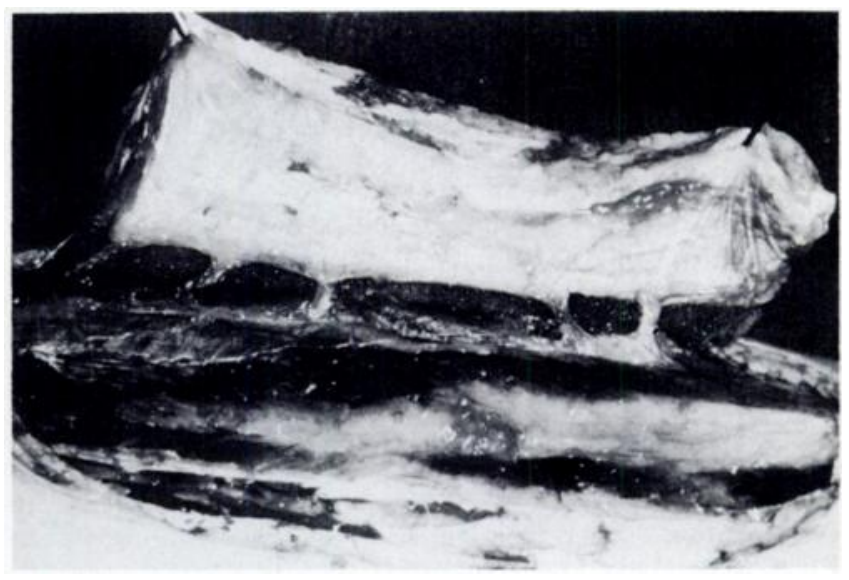

Fig. 3

Cutaneous flap raised. showing its dependence on the cutaneous branches of the peroneal artery. 


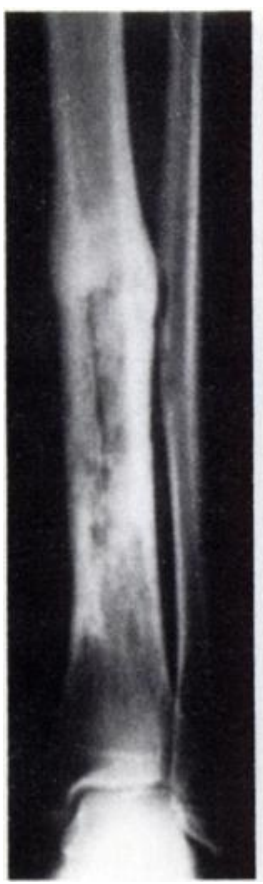

Fig. 4

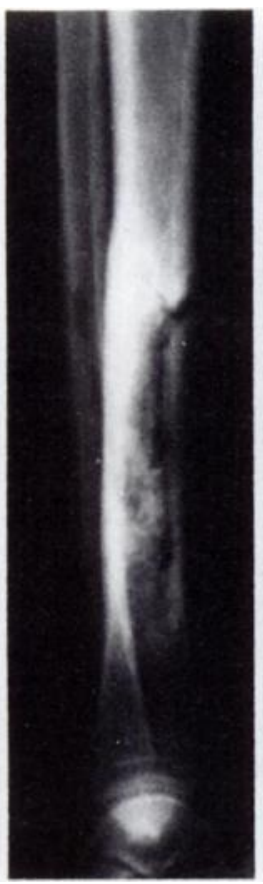

Fig. 5

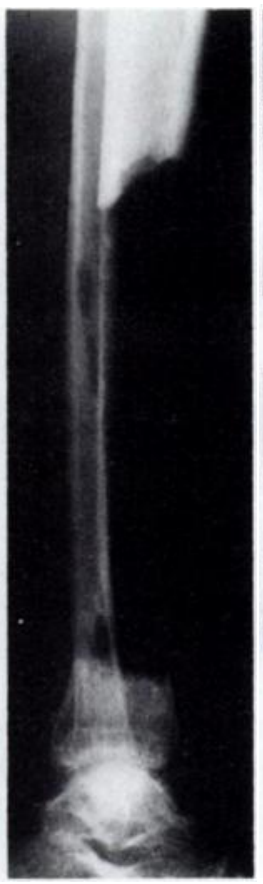

Fig. 6

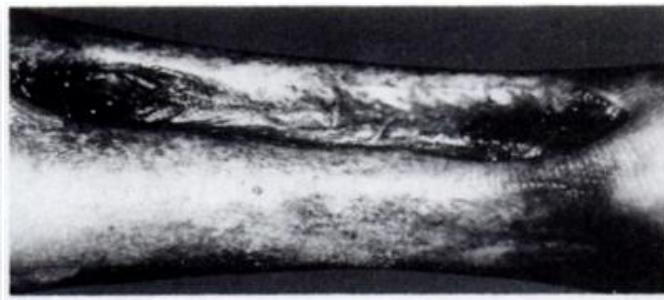

Fig. 7

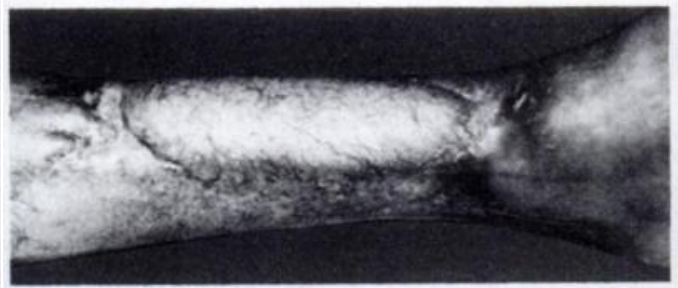

Fig. 8

Case 1. Figures 4 and 5-Radiographs showing extensive osteonecrosis and infection. Figure 6-After resection, showing the large bony defect. Figure 7 Epithelialised skin defect over the tibia before flap transfer. Figure 8 - Flap soundly healed despite old radiation damage to the surrounding skin. Figure 9 After grafting, showing union with tibia and talus.

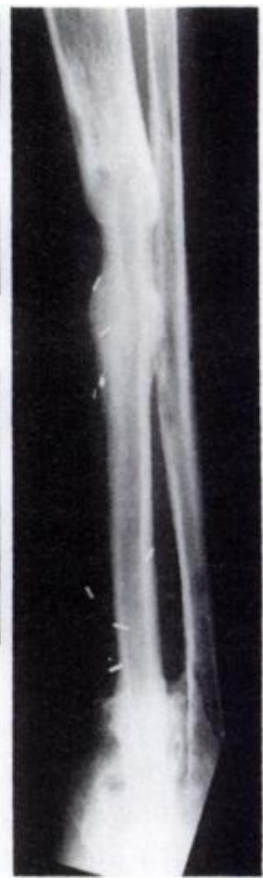

Fig. 9

\section{CASE REPORTS}

Case 1. A 20-year-old man presented with a history that at the age of 13 a Ewing sarcoma of his left tibia had been treated with heavy irradiation. and later with cylotoxic drugs. For two years he had had a discharging sinus in the lower mid-shaft region, surrounded by skin showing marked radiation damage. A radiograph showed an extensive area of osteomyclitis with sclerosis and cavity formation (Figs 4 and 5).

At operation a $13 \mathrm{~cm}$ segment of the lower tibia was removed and the medullary cavity curetted both proximally and distally. The resulting defect was skin-grafted. Healing was not at first satisfactory and further removal of bone was required (Fig. 6). Eventually epithelialisation was achieved and it was then assumed that the infection had been cleared and all dead bone removed. The distal tibial segment, however, was so short and osteoporotic that it was considered unlikely that bony union could be achieved at this end.

A free osteocutaneous fibular graft was raised from the opposite leg. The bone graft was $18 \mathrm{~cm}$ long and extended $5 \mathrm{~cm}$ distally beyond the end of the skin flap. The vascularity of this bone end was confirmed. The distal end of the bone graft was inserted through the distal tibial fragment and across the ankle joint into the talus after the removal of articular cartilage. Postoperative recovery was satisfactory and the skin flap healed well apart from some delay at the proximal end (Figs 7 and 8). Eighteen months after operation the patient was able to walk without support (Fig. 9)

Case 2. A 35-year-old man lost $13 \mathrm{~cm}$ of his lower tibia in a road traffic accident. After debridement the area was skin-grafted. being held out to length by the intact fibula. A radiograph showed some early new bone formation from the posterior periosteum but not enough to allow weight-bearing. An osteocutaneous free vascularised fibular graft was therefore raised from the opposite leg with a skin flap $15 \mathrm{~cm}$ by $7 \mathrm{~cm}$. The fibular graft was inset into the medullary cavity of the tibia both proximally and distally and the graft revascularised, while the donor site was skin-grafted. Postoperatively there was again a small proximal area of necrosis in the flap but this healed satisfactorily and the graft united and thickened (Figs 10 and II).

Other cases. Seven osteocutaneous fibular transfers have been successfully completed. The average length of the bone graft was $14 \mathrm{~cm}$ with a range of $131018 \mathrm{~cm}$. The width of the skin flap ranged from $71013 \mathrm{~cm}$. and there was no major loss of skin. Four of the patients can now walk unsupported by any prosthetic appliance. Three show union but are protected from fracture of the fibular graft by light-weight calipers and will remain so for 18 months postoperatively.
Fig. 10

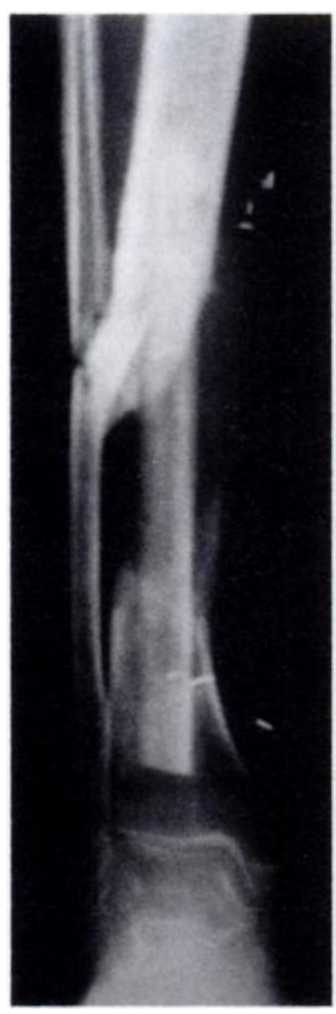

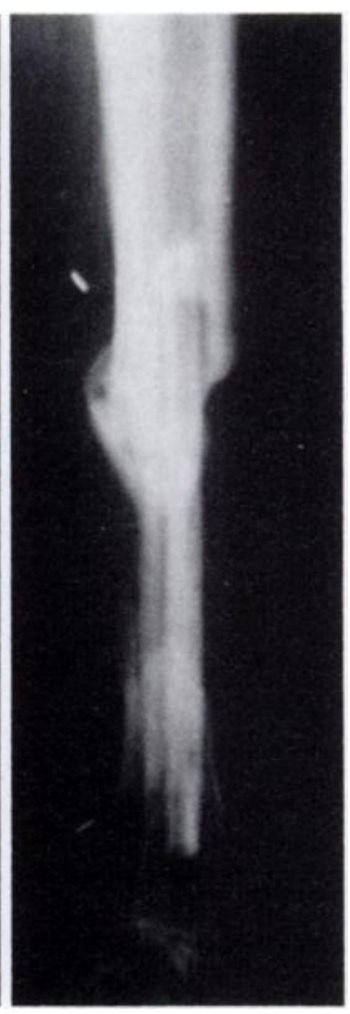

Fig. 11
Case 2. Radiographs to show a free vascularised fibular graft united to the tibia at both ends. 


\section{DISCUSSION}

An osteocutaneous free fibular graft is relatively easy to take, provided that the principles of its cutaneous circulation are fully understood. The segmental distribution of the cutaneous vessels (see Figs 2 and 3 ) does tend to place the most proximal branch at risk; this may be the cause of the occasional small proximal area of necrosis. This branch stems from the peroneal artery fairly close to its own origin and great care is needed to preserve it when freeing the proximal end of the peroneal artery from the flap.

The one disadvantage of the osteocutaneous flap then becomes apparent, namely that it is rare to have sufficient length of peroneal artery to permit end-to-side anastomosis to a recipient vessel. It is usually necessary to use an end-to-end anastomosis with the consequent risk to the distal circulation and the chance of spasm in the recipient vessel. Vein grafts can be used to elongate the vessels and permit end-to-side anastomosis. The flap itself, however, has proved reliable and it is thin, making it much easier to inset than the often fat groin skin carried with a deep circumflex iliac artery flap.

The use of a cutaneous flap with the free fibular graft allows estimation of the circulatory state either visually or by the use of the photoplethysmograph, more valuable in our experience than technetium bone scans in assessing bone vascularisation. The fixation of the bone graft at operation is made relatively easy by insetting it into the medullary cavities, though often the presence of an intact fibula in the recipient leg may restrict the manipulation needed to insert a length of donor fibula. In placing the fibular graft care must also be taken to avoid damage to the flap artery. One of the most effective ways of moving the graft is to drill a hole into its cortex and then use a bone hook. When the bone graft has been placed into the shaft, metal fixation is unnecessary. In the postoperative period support is provided either with an external fixator or with an above-knee plaster cast for about five months. It is often not easy to confirm radiological union even after clinical union is apparent.

Fibular grafts may fracture; this occurs in the midshaft region after union at both ends and protection with a caliper or a Sarmiento-type of cast is therefore indicated for an extended period. Should fractures occur they are usually undisplaced cracks and the subperiosteal new bone formation that occurs tends to increase the girth of the graft more quickly. It is usually 18 months after the operation before the fibular grafts are ready to take unprotected weight-bearing.

Conclusions. The relatively easy and rapid availability of a viable skin flap together with a vascularised fibular graft has made it possible to treat patients with massive defects in long bones by a one-stage procedure. The value of the fibular graft has already been successfully confirmed, and the associated skin flap is reliable.

\section{REFERENCES}

Buncke HJ, Furnas DW, Gordon L, Achauer BM. Free osteocutaneous flap from a rib to the tibia. Plast Reconstr Surg 1977:59:799 805.

Chen $\mathbf{Z W}$, Yan $\mathbf{W}$. The study and clinical application of the osteocutaneous flap of fibula. Microsurgery 1983:4:11 6.

Gilbert A. Vascularized transfer of the fibular shaft. Int $J$ Microsurg 1979:1:1002.

Haw CS, O'Brien BMcC, Kurata T. The microsurgical revascularisation of resected segments of tibia in the dog. J Bone Joint Surg [Br] 1978:60 B:266- 9.

Tamai S, Sakamoto H, Hori $Y$, et al. Vascularized fibula transplantation: a report of 8 cases in the treatment of traumatic bony defect or pseudarthrosis of long bones. Int J Microsurg 1980:2: $205-12$.

Taylor GI, Miller GDH, Ham FJ. The free vascularized bone graft: a clinical extension of microvascular techniques. Plast Reconstr Surg 1975:55:533 44.

Taylor GI, Watson N. One-stage repair of compound leg defects with free vascularized flaps of groin skin and iliac bone. Plast Reconstr Surg 1978:61:494-506. 